\title{
3D Confocal Fluorescence Microscopy Analysis of Skeletal Muscle Myogenesis in Self Assembled 3D Microtissues
}

\author{
Gianna P. Ahnrud ${ }^{1 *}$, Jeffrey Morgan ${ }^{2}$, Kevin O'Fallon $^{1}$, Patrick Marek ${ }^{1}$ \\ 1. Combat Capabilities Development Command - Soldier Center, Natick, MA, USA. \\ 2. Department of Molecular Pharmacology, Physiology \& Biotechnology Brown University, Providence, \\ RI, USA. \\ * Corresponding author: gianna.p.ahnrud.civ@mail.mil
}

Traditional monolayer 2D cell culture and 2D in vitro assays for drug development have their limitations related to human physiological relevance. Efforts to expand on 2D models and reduce the use of animals in research have led to progressive developments in 3D cell culture systems as tools for drug discovery [1]. These tools provide a platform for enhanced cell to cell interaction and cellular differentiation [1]. A variety of 3D platforms exist including collagen or other biomimetic scaffolding, synthetic scaffolds, and hydrogels to promote cell self-assembly [2-4]. A non-adherent series of 3D agarose gels has been developed by the Morgan Lab of Brown University and demonstrates success in promoting cell selfassembly into 3D microtissues. Successful characterization of skeletal muscle myogenesis in a 3D in vitro model is important to establish a baseline for future drug discovery in the medical community and nutritional intervention strategies by the military to mitigate muscle damage. 3D confocal fluorescence imaging analysis demonstrated the ability of the Morgan Lab 3D Petri Dish $[3,4]$ to induce myogenesis, the process of skeletal muscle growth and differentiation in $\mathrm{C} 2 \mathrm{C} 12$ mouse skeletal muscle cells [5]. $\mathrm{C} 2 \mathrm{C} 12$ cells were grown to confluency in $775 \mathrm{~cm}^{2}$ cell culture flasks at $37^{\circ} \mathrm{C}, 10 \% \mathrm{CO}_{2}$, trypsinized and seeded into $2 \%$ agarose/PBS hydrogels and allowed to self-assemble into toroid shaped microtissues (Fig. 1). Initial microtissue formation was assessed using a Zeiss brightfield microscope. Microtissues were formalin fixed at day 1,3 and 6 then skeletal muscle myogenesis and cellular differentiation was determined by fluorescent antibody staining for myosin heavy chain protein. Confocal images were obtained using the Perkin Elmer Opera Phenix High Content Screening System and Image processing was performed using 3D Harmony High Content Imaging and Analysis Software.

Brightfield microscopy confirmed that $\mathrm{C} 2 \mathrm{C} 12$ cells formed toroid shaped microtissues in Advanced DMEM supplemented with Glutamax (Fig. 1). Myosin heavy chain protein expression was detected on day 1 while multinucleation and myofiber formation were visible as early as 3 days after cell seeding (Fig 2a).

Advances in 3D confocal fluorescence microscopy with automated $\mathrm{Z}$ stack imaging and high throughput image analysis software allowed for thorough investigation of myogenesis in $\mathrm{C} 2 \mathrm{C} 12$ toroid microtissues (Fig. 2b). These analysis capabilities are necessary and effective for the validation of new in vitro model platforms such as the 3D Petri Dish toroid agarose mold to successfully demonstrate their abilities to modulate in vivo like characteristics in 3D microtissues [6].

\section{References:}

[1] S. Breslin and L. O’Driscoll, Drug Discov. Today 18 (2013), p. 240-249,.

[2] M. Beldjilali-Labro et al., Materials (Basel) 11 (2018).

[3] B. T. Wilks, E. B. Evans, M. N. Nakhla, and J. R. Morgan, Acta Biomater., (2018). 
[4] T. M. Achilli, S. McCalla, J. Meyer, A. Tripathi, and J. R. Morgan, Mol. Pharm. 11 (2014), p. 2071.

[5] A. Musarò, Adv. Biol. 2014 (2014), p. 1.

[6] Acknowledgements: Mr. Joshua Magnone and Mr. Kenneth Racicot CCDC-Soldier Center, DoD SMART Program
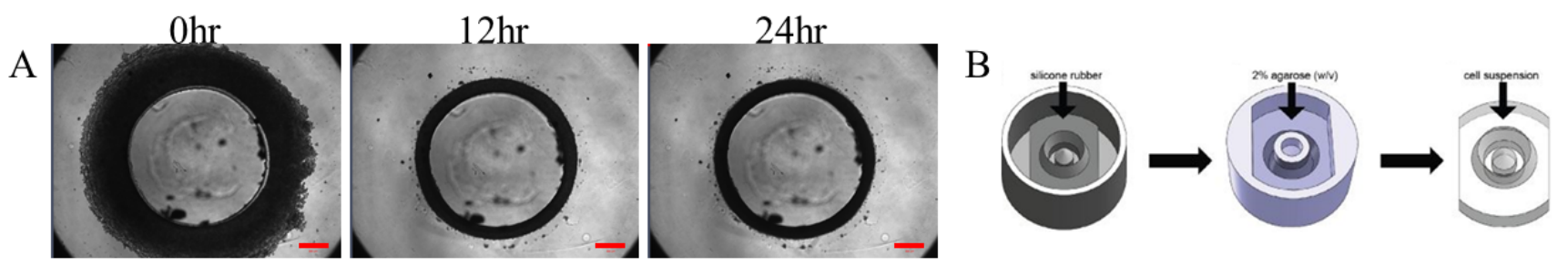

Figure 1. A) Brightfield imaging of $\mathrm{C} 2 \mathrm{C} 12$ cells forming microtissues over time. Scale bar $=200 \mu \mathrm{m}$. B) Morgan Lab 3D Petri Dish design from Wilks et al 2018[3].
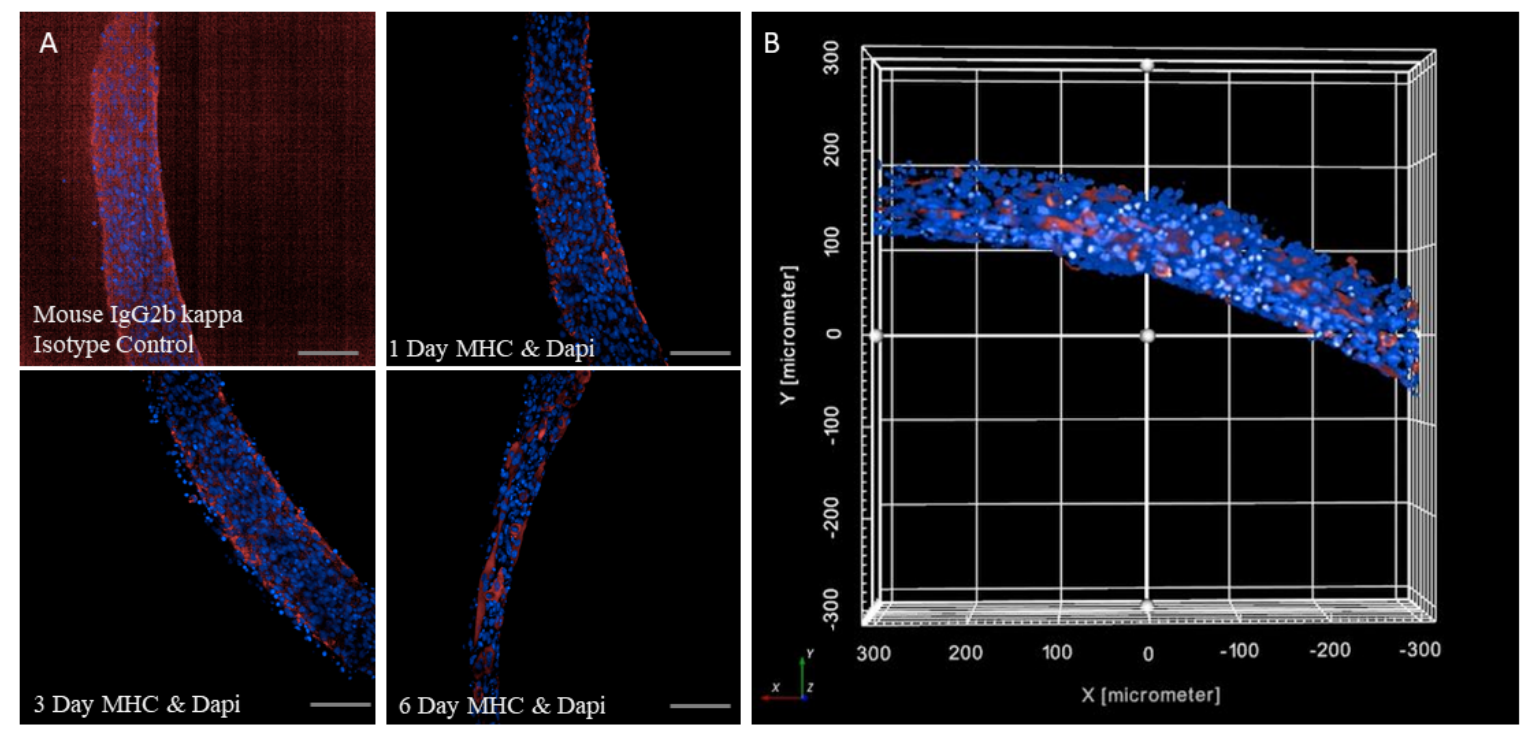

Figure 2. A) Confocal imaging demonstrates $\mathrm{MHC}$ (red) expression and Dapi (blue) in C2C12 toroid microtissues over time. Images were acquired on the Opera Phenix High Content Screening System with a 20x water objective. Scale bar $=100 \mathrm{um}$. B) 3D Harmony High Content Imaging Analysis Software provides $3 \mathrm{D} Z \mathrm{Z}$ stack rendering of a segment of Day $6 \mathrm{C} 2 \mathrm{C} 12$ microtissue displaying toroid differentiation. 